\title{
A bright coronal downflow seen in multi-wavelength observations: evidence of a bifurcating flux-rope ?^
}

\author{
D. Tripathi ${ }^{1}$, S. K. Solanki ${ }^{2}$, H. E. Mason ${ }^{1}$, and D. F. Webb ${ }^{3}$ \\ 1 Department of Applied Mathematics and Theoretical Physics, Wilberforce Road, Cambridge CB3 0WA, UK \\ e-mail: [D.Tripathi;H.E.Mason] @damtp.cam.ac.uk \\ 2 Max-Planck-Institut für Sonnensystemforschung, 37191 Katlenburg-Linda, Germany \\ e-mail: solanki@mps.mpg.de \\ ${ }^{3}$ Institute for Scientific Research, Boston College, Chestnut Hill, Massachusetts, USA \\ e-mail: David.Webb.ctr@hanscom.af.mil
}

Received 24 April 2007 / Accepted 2 July 2007

\begin{abstract}
Aims. We study the origin and characteristics of a bright coronal downflow seen after a coronal mass ejection associated with erupting prominences on 5 March 2000.

Methods. This study extends that of Tripathi et al. (2006b, A\&A, 449, 369) based on the Extreme-ultraviolet Imaging Telescope (EIT), the Soft X-ray Telescope (SXT) and the Large Angle Spectrometric Coronagraph (LASCO) observations. We combined those results with an analysis of the observations taken by the $\mathrm{H} \alpha$ and the Mk4 coronagraphs at the Mauna Loa Solar Observatory (MLSO). The combined data-set spans a broad range of temperature as well as continuous observations from the solar surface out to $30 R_{\odot}$. Results. The downflow started at around 1.6 $R_{\odot}$ and contained both hot and cold gas. The downflow was observed in the H $\alpha$ and the Mk4 coronagraphs as well as the EIT and the SXT and was approximately co-spatial and co-temporal providing evidence of multi-thermal plasma. The $\mathrm{H} \alpha$ and Mk4 images show cusp-shaped structures close to the location where the downflow started. Mk4 observations reveal that the speed of the downflow in the early phase was substantially higher than the free-fall speed, implying a strong downward acceleration near the height at which the downflow started.

Conclusions. The origin of the downflow was likely to have been magnetic reconnection taking place inside the erupting flux rope that led to its bifurcation.
\end{abstract}

Key words. Sun: corona - Sun: coronal mass ejections (CMEs) - Sun: prominences - Sun: filaments

\section{Introduction}

Observations of coronal downflows in X-rays (McKenzie \& Hudson 1999; McKenzie 2000), EUV radiation (Innes et al. 2003a,b; Asai et al. 2004) and in white-light (Wang et al. 1999; Sheeley \& Wang 2002) after Coronal Mass Ejections (CMEs) were first detected based on observations made by the Soft X-ray Telescope (SXT; Tsuneta et al. 1991) aboard Yohkoh, the Transition Region and Coronal Explorer (TRACE; Handy et al. 1999) and the Solar Ultraviolet Measurements of Emitted Radiation (SUMER; Wilhelm et al. 1995) spectrometer and the Large Angle Spectrometer Coronagraph (LASCO; Brueckner et al. 1995) aboard the Solar and Heliospheric Observatory (SoHO; Domingo et al. 1995), respectively. These downflows appeared to be dark and were interpreted as plasma voids with high temperature and low density as a consequence of magnetic reconnection following the CME eruptions.

Recently, Tripathi et al. (2006b), referred to as "Paper I" hereinafter, reported a bright coronal downflow after a CME event, which occurred on 5 March 2000. This was the first bright coronal downflow following a CME eruption observed at EUV wavelengths by the Extreme-ultraviolet Imaging Telescope (EIT; Delaboudiniere et al. 1995; Moses et al. 1997) also aboard the SoHO. Based on EIT 304 Å observations de Groof et al. (2004) presented bright coronal downflows in a coronal loop without any associated eruption. The origin of these downflows was explained by numerical simulations of "catastrophic cooling" in a coronal loop which is heated predominantly at its footpoints (de Groof et al. 2005).

In contrast to the dark downflows following eruption, the bright downflow indicates a flow of heated plasma, thus providing more direct evidence of magnetic reconnection during the eruption of CMEs. The corresponding CME was associated with three erupting prominences. Based on the analysis of the images obtained by EIT at $195 \AA$ and by SXT, it was speculated that the downflow could indeed be a consequence of magnetic reconnection, taking place somewhere outside the field-of-view (FOV) of EIT, but behind the occulter of the LASCO/C2. Figure 1 displays the running difference images of the downflow taken by the EIT at $195 \AA$. The fact that the downflow started in the gap between the area covered by two instruments leads to significant uncertainties in the interpretation. We have therefore searched other databases with the aim of finding data covering this uncharted region. This event was fortuitously also recorded by the $\mathrm{H} \alpha$ coronagraph, the Mk4 coronameter and the He $10830 \AA$ telescope at Mauna Loa Solar Observatory $\left(\mathrm{MLSO}^{1}\right)$ in Hawaii. These telescopes nicely fill the gap between the EIT and the LASCO C2.

^ Movies are available in electronic form at http: //www . aanda.org

${ }^{1}$ http://mlso.hao.ucar.edu/cgi-bin/mlso_homepage.cgi 

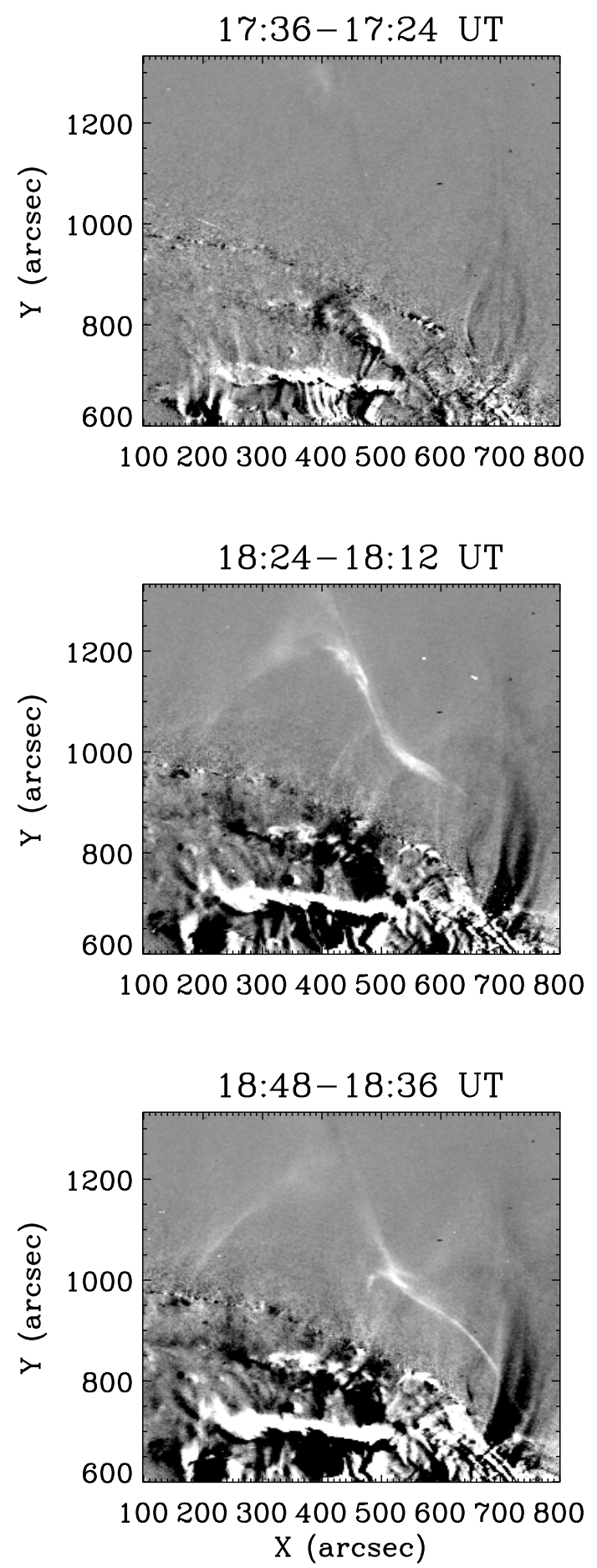

Fig. 1. Running difference images taken by EIT at $195 \AA$ on 5 March 2000 showing the bright coronal downflow. In the top image it is just visible at the top of the panel at $x \approx 400$ arcsec.

While MLSO did not catch the eruption phase, it did provide observations of the downflow.

Observations of downflows in the majority of erupting prominences were reported by Gilbert et al. $(2000,2001)$ based on the data taken with HAO's (High Altitude Observatory) MLSO instruments. According to Gilbert et al. (2000, 2001), in the process of eruption the prominences break into two parts involving the formation of an X-type neutral line and magnetic reconnection. The separation seemed to take place at a height ranging from 1.20 to $1.35 R_{\odot}$. However, there was no direct evidence for magnetic reconnection and the formation of an Xtype neutral line. Moreover, no attempts were made to compare the $\mathrm{H} \alpha$ observations with data taken by other instruments such as SoHO/EIT or Yohkoh/SXT.

Based on the observations made by the $\mathrm{H} \alpha$ coronagraph at MLSO, Gilbert et al. (2001) discussed that, for the flux-rope type of topology, reconnection could take place either below or within the flux rope during the eruption. Generally, in "standard 2D models" it is considered that the magnetic field lines overlying the flux-rope reconnect at the current sheet in the wake of expulsion of the flux-rope (e.g., Lin \& Forbes 2000, and references therein). In this scenario the total expulsion of the flux rope occurs and the flux rope propagates into the interplanetary medium along with the corresponding CME. Note that in this scenario the magnetic field lines forming the flux rope do not take part in reconnection. On the other hand, the reconnection could also take place "internally", i.e., within the flux rope where field lines forming the flux rope take part in reconnection (Manchester et al. 2004; Gibson \& Fan 2006a,b), leading to the bifurcation of the flux rope during eruption.

The observations and hypothesis made by Gilbert et al. (2000, 2001) were later studied by Gibson \& Fan (2006a,b) based on a 3D MHD simulation. In these simulations the complete evolution of a flux rope was studied from the solar surface out to $6 R_{\odot}$. Based on the results of their simulation, Gibson $\&$ Fan $(2006 a, b)$ found that the emergence of a flux rope with enough twist causes it to erupt due to loss of equilibrium. The flux rope undergoes a kink instability which leads to the formation of a vertical current sheet inside the flux rope. The formation of a current sheet within an unstable flux rope has also been demonstrated by Birn et al. (2006). After multiple reconnections occurring inside the flux rope at the current sheet, formed during the eruption, the flux-rope breaks in two. One part of the flux rope escapes as the core of a corresponding CME and the other falls back towards the Sun's surface. However, in the simulation presented by Gibson \& Fan (2006a,b), a special kind of magnetic field geometry was used - a Bald Patch Separatrix Surface (BPSS) - where the flux-rope intersects the photosphere and thus there is no X-point below the flux-rope.

In this paper we present observations of a bright coronal downflow which may be an example of such a bifurcating flux rope in the course of a CME eruption. Here we investigate the observation of the downflow taken in multiple wavelengths, such as $\mathrm{H} \alpha$, white-light $\mathrm{K}$-corona (Mk4), and EUV. In the next section, we present the observations we used, followed by their analysis and results in Sect. 3. In Sect. 4, we provide measurements performed on the data. We provide a summary of the results and discussion in Sect. 5.

\section{Observations}

Three erupting prominences (two large and one small - barely discernible) followed by a coronal downflow were observed on 5 March 2000 by the EIT. The EIT provides observations of the Sun at $195 \AA$ with a regular cadence of about 12 min and one image every $6 \mathrm{~h}$ at $171 \AA$, $284 \AA$ and $304 \AA$. The $195 \AA$ passband of EIT is dominated by an Fe XII line formed at $1.5 \mathrm{MK}$, but also contains an Fe XXIV line at $192 \AA$ formed at around $20 \mathrm{MK}$, which is usually much weaker in the quiet Sun region but highly significant in flaring regions (Tripathi et al. 2006a). The images obtained by the EIT at $171 \AA$, $284 \AA$ and $304 \AA$ wavelengths are dominated by lines Fe IX/x (1.0 MK), Fe XV (1.8 MK) and He II $(0.05 \mathrm{MK})$ respectively. 

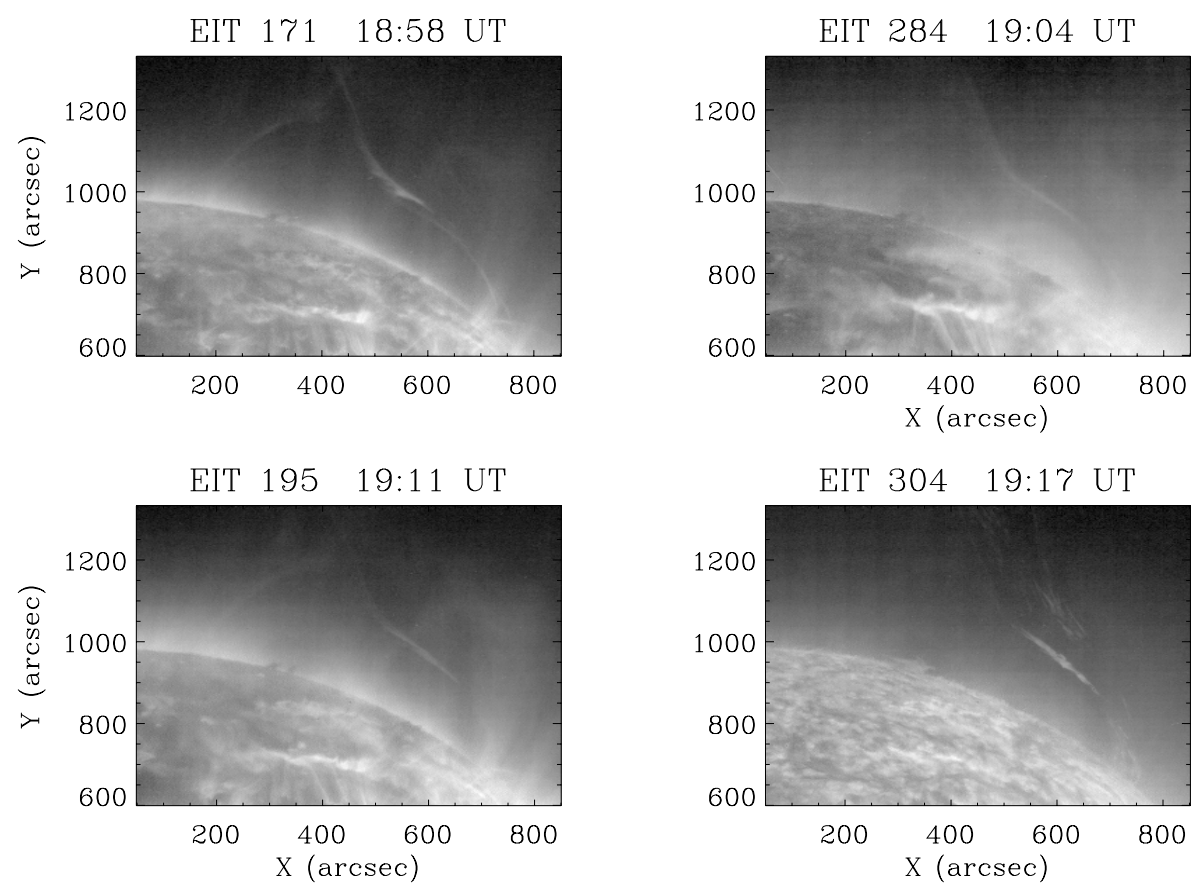

Fig. 2. Absolute intensity images recorded by the EIT in its $171 \AA$ (top left), $284 \AA$ (top right), $195 \AA$ (bottom left) and $304 \AA$ (bottom right) channels. Note that the images are displayed on a logarithmic scale.

Fortuitously this downflow was also observed by the Advanced Corona Observing System (ACOS) ${ }^{2}$ composed of the Polarimeter for Inner Coronal Studies (PICS) H $\alpha$ (6563 A) coronagraph, the Mk4 K-coronameter, which observes the whitelight K-corona, and the Chromospheric Helium I Imaging Photometer (CHIP) He I (10830 ̊) instrument. In this paper we concentrate on observations taken by the $\mathrm{H} \alpha$ coronagraph and the Mk4 coronameter. Since the downflow was not clearly seen in the CHIP data, we decided not to use it.

The ACOS instruments are operated at MLSO in Hawaii by the HAO. They operate every day from about 17:00 until 22:00 UT (weather permitting) producing about 100 images for each instrument with a cadence of about $3 \mathrm{~min}$. The field of view (FOV) ranges from 1.01 to $1.83 R_{\odot}$ from the solar center for the $\mathrm{H} \alpha$ coronagraph and from 1.12 to $2.79 R_{\odot}$ for Mk4. The pixel size of the images taken by the $\mathrm{H} \alpha$ coronagraph and Mk4 coronameter is about 2.9 arcsec. For white-light observations of the corona, the $\mathrm{Mk} 4$ coronameter records the polarization brightness data as well as white-light vignetted data, where a hypothetical density function is subtracted from the actual data in order to enhance the contrast in the images. Since we are interested in a morphological study, we consider the Mk4 white-light vignetted data in this paper. The Mk4 data provided in the archive are essentially fully processed. For technical details concerning data processing and the instruments see Elmore et al. (2003).

\section{Data analysis and results}

About 90 min (at 17:36 UT) after the onset of the eruption, a bright feature appeared at the edge of the FOV of the EIT. After a while, this bright feature propagated towards the Sun's surface and is referred to as the bright coronal downflow. Figure 1 displays a series of running difference images taken by the EIT at $195 \AA$, revealing the morphological evolution of the downflow in time. Since the EIT observations are described in detail in Paper I, we restrict ourselves to saying that the three images

\footnotetext{
${ }^{2}$ http://mlso.hao.ucar.edu/cgi-bin/ mlso_data.cgi?2000\&ACOS
}

show from top to bottom the initial appearance of the downflowing material at the edge of the EIT FOV, an intermediate phase and a late phase of the downflow respectively. EIT also recorded one image using $171 \AA$, $304 \AA$ and $284 \AA$ channels in the very late phase of the downflow (see Fig. 2). These images reveal a multi-temperature behaviour of the downflowing plasma. Since the EIT provided only one image during the downflow phase using these channels, we could not study the evolution of the downflow plasma in other channels.

Figure 3 displays a sequence of images taken by the $\mathrm{H} \alpha$ coronagraph. The first image was taken at 17:09 UT. Although the ACOS instruments recorded the very early phase (start phase) of the downflow, they unfortunately missed the eruption phase. The FOV of the $\mathrm{H} \alpha$ observation is ideal for an investigation of the origin and evolution of this downflow. Although, the $\mathrm{H} \alpha$ coronagraph provides data with a regular cadence of about three minutes, we only show some selected images in Fig. 3. For the complete sequence in animation format see movie "halpha.mov"3. At 17:09 UT (top left frame in Fig. 3) a lot of material is piled high up in the corona with some bright threads still connected to the Sun's surface. As time passes, some of the material seems to move away and might have escaped along with the CME. In addition some material moves downward.

After a while (at 17:48 UT) multiple bright localized structures (cusp-shaped features) can be seen at the location (950, 1200 ; in arcsec). These localized features are marked as "S1", "S2" and "S3". The feature on the right, namely "S1", is brightest. These three features exist until 17:54 UT. After that only one, very bright (brighter than the earlier three) cusp-shaped feature remains, which then propagates downward. The two branches in the downflow, marked "B1" and "B3", emanate from this cusp and material flows down along these two branches before the right branch "B1" bifurcates into another branch namely "B2" at 18:07 UT. Interestingly this location and time corresponds to the kink in the right branch seen in the EUV images (see Fig. 1). The two branches "B1" and "B3" of the downflow are also evident in the EUV observations, though the left "B3"

\footnotetext{
3 The movies are available online.
} 

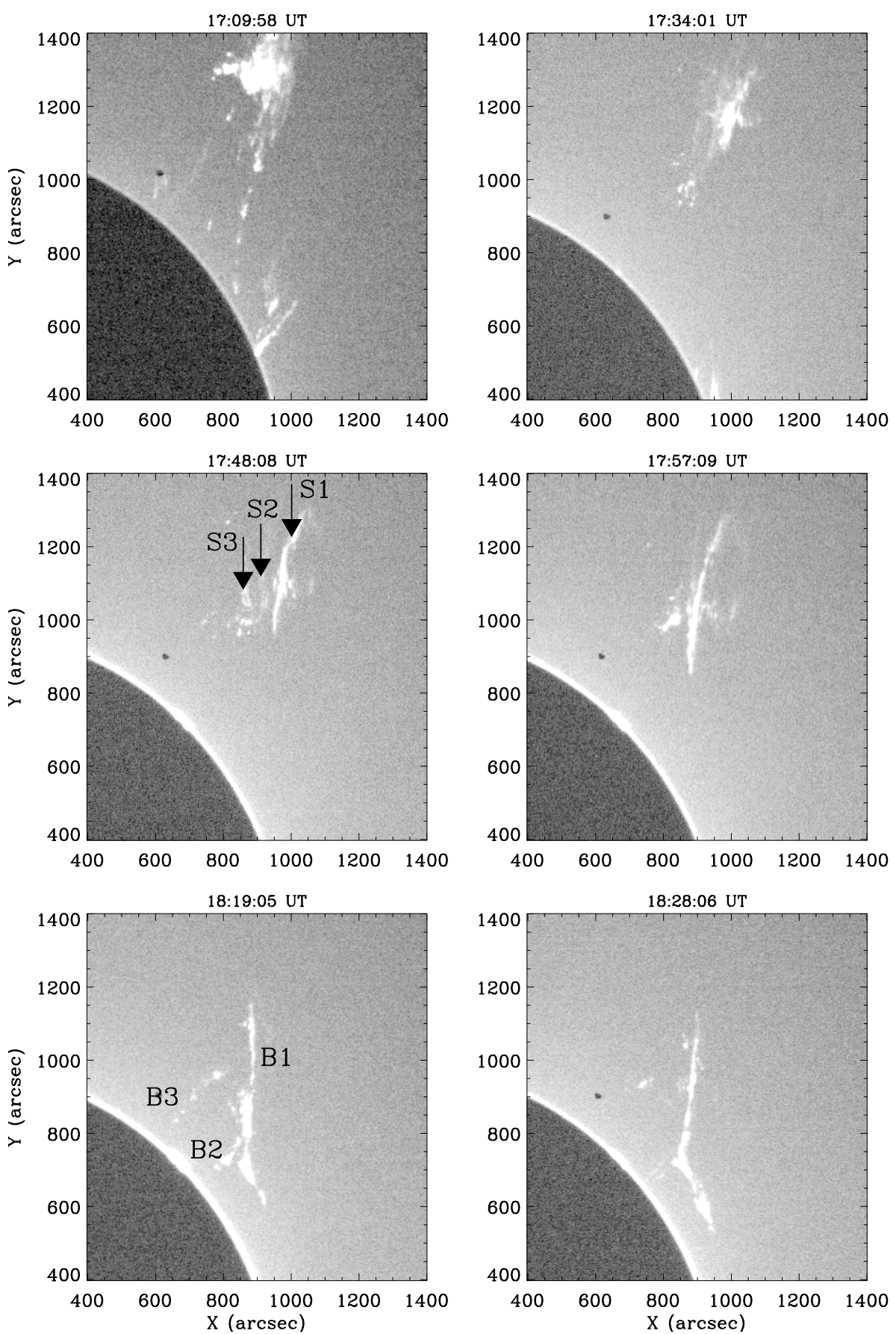

Fig. 3. Sequence of images taken by the $\mathrm{H} \alpha$ coronagraph of the Advanced Corona Observing System at Mauna Loa Solar Observatory showing the complete morphology of the downflow. The images are displayed on a logarithmic scaling of intensity. Note that in this figure only some selected images are shown. For the complete evolution of the downflow see the movie "halpha.mov" (online only).

branch is not as clearly discernible as in $\mathrm{H} \alpha$ observations. The third branch of the downflow "B2", which is bright and strong in $\mathrm{H} \alpha$ images, is not evident in the EUV images, most likely because the plasma flowing along the third branch does not radiate in the narrow temperature range to which the EIT $195 \AA$ channel is sensitive. The left branch ("B3") had almost disappeared by 18:37 UT and the middle branch ("B2") disappears at 18:28 UT. However, the kink in the right branch - where the middle branch emanates - remains clearly visible. Most of the material seems to flow down along the right branch of the downflow feature, which is the brightest and longest lasting.

In order to compare our EIT observations with those made in $\mathrm{H} \alpha$, we display in Fig. 4 three EIT images co-aligned with $\mathrm{H} \alpha$ images recorded very close in time (top panel: EIT17:10:48 UT, $\mathrm{H} \alpha-17: 09: 58 \mathrm{UT}$; middle panel: EIT-17:58:50 UT,
H $\alpha$-17:57:09 UT; bottom panel: EIT-18:23:03 UT, H $\alpha$ 18:23:06 UT). Since the $\mathrm{H} \alpha$ coronagraph does not provide full disk observations like EIT, it is not straightforward to co-align these images. For the co-alignment, we co-registered the EIT images to the near-simultaneous $\mathrm{H} \alpha$ images using the routine coreg_map.pro provided in the SSW tree ${ }^{4}$. This routine is a wrapper around another routine called drot_map.pro which differentially rotates one map at the time of the other map, while taking into account the roll angle. Also, in order to have same pixel size in two maps, images with a smaller pixel size are rebinned to a high number of pixels. The co-registration provides the EIT images with the same pixel size $(2.9$ arcsec $)$ as that of $\mathrm{H} \alpha$ images. The $\mathrm{H} \alpha$ contours representing the right branch of the downflow (right panel of Fig. 4) were seen to be spatially and

\footnotetext{
${ }^{4}$ http://ydac.mssl.ucl.ac.uk/sswdoc/solarsoft/
} 

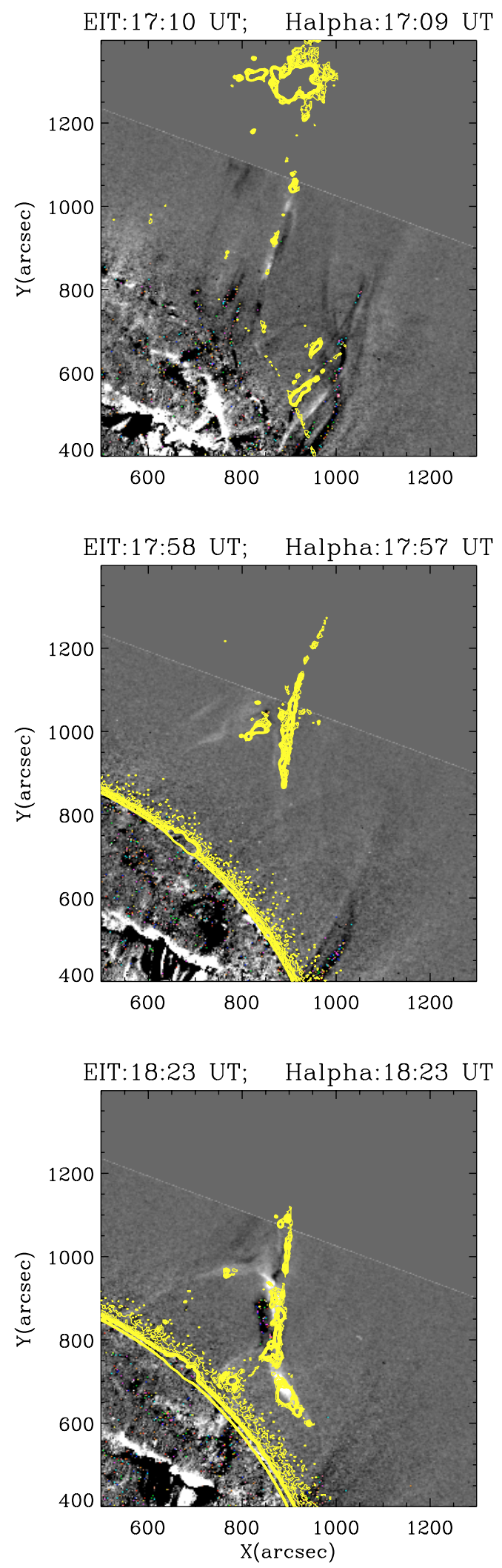

Fig. 4. Co-aligned over-plotted EIT (running difference; in black \& white) and $\mathrm{H} \alpha$ images (yellow contours).

temporally correlated with those of EIT. In the left branch the $\mathrm{H} \alpha$ brightenings appear to be far more localized and point-like than the more thread-like structures seen in EIT. These bright $\mathrm{H} \alpha$ points do, however, correspond to EIT brightenings. It seems
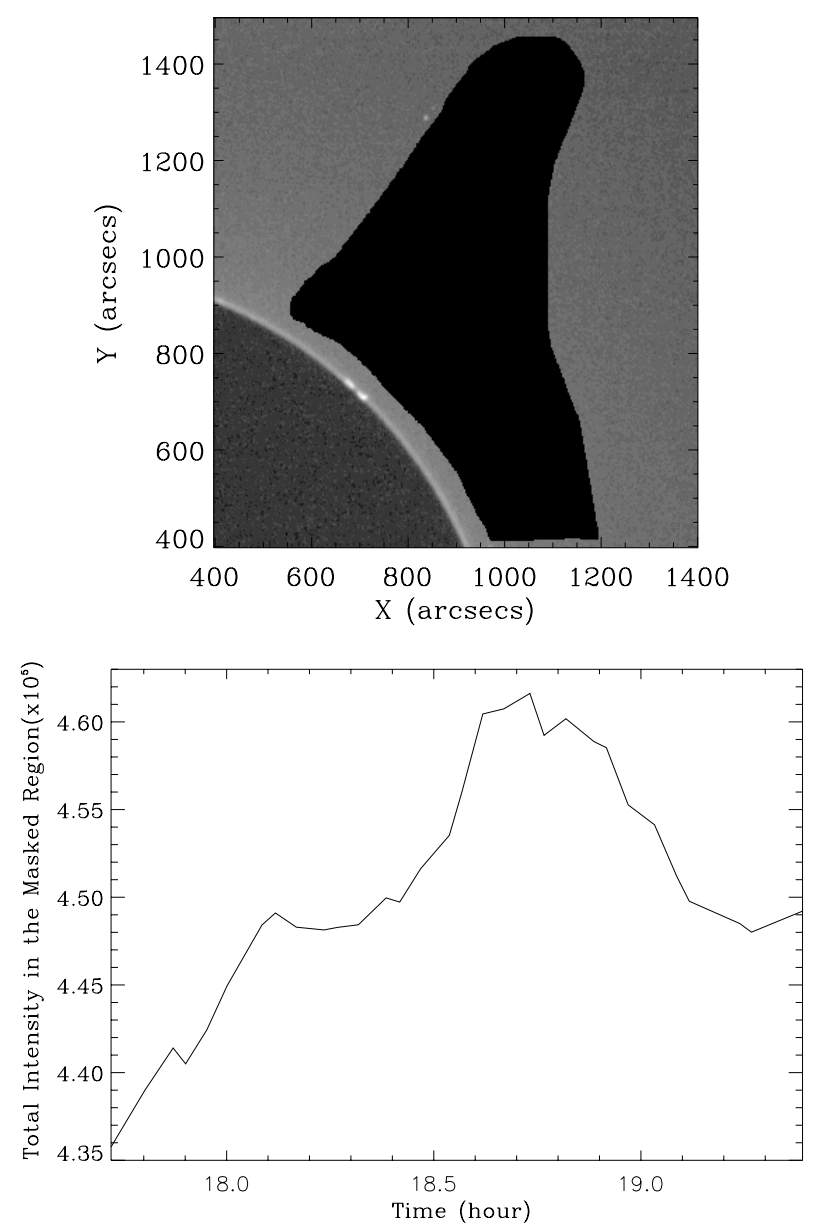

Fig. 5. Top panel: masked $\mathrm{H} \alpha$ image. Bottom panel: variation of total intensity in the masked region with time.

plausible and reasonable to conclude that the features observed in EIT and those in $\mathrm{H} \alpha$ are closely connected, although due to different temperature sensitivity of the 2 data sets it is likely that the plasma is composed of multi-thermal unresolved magnetic strands. The cusp-shaped feature seen in $\mathrm{H} \alpha$ is located outside the FOV of EIT. Moreover, downflow branches in the EIT and the apex location where the two branches emanate in EUV images seem to be wider than that in $\mathrm{H} \alpha$.

The top panel of Fig. 5 displays an $\mathrm{H} \alpha$ map with a masked region. We selected this region in order to compute the variation of the total amount of material during the sequence of the downflow which is shown in the bottom panel. It is evident from the plot (see bottom panel of Fig. 5) that the total intensity of the masked region increases over most of the time that the downflow was seen. This could either be due to the increase in the amount of the downflowing plasma, to an enhancement of the density, or cooling of hot material to chromospheric temperatures. Further the total intensity starts to decrease when most of the material has drained down on to the Sun's surface.

The downflow was also recorded in white-light observations taken by the Mk4 coronameter of the ACOS at MLSO. The advantage of Mk4 is the larger FOV. Combining the EIT, the $\mathrm{H} \alpha$ and the Mk4 observations provides an opportunity to study the solar corona out to $2.79 R_{\odot}$. Since white-light images can be directly interpreted in terms of the distribution of electron density along the line of sight, they potentially contain information on the dynamics of the magnetic field. Figure 6 displays absolute intensity images taken by the Mk4 coronagraph. Three bright 

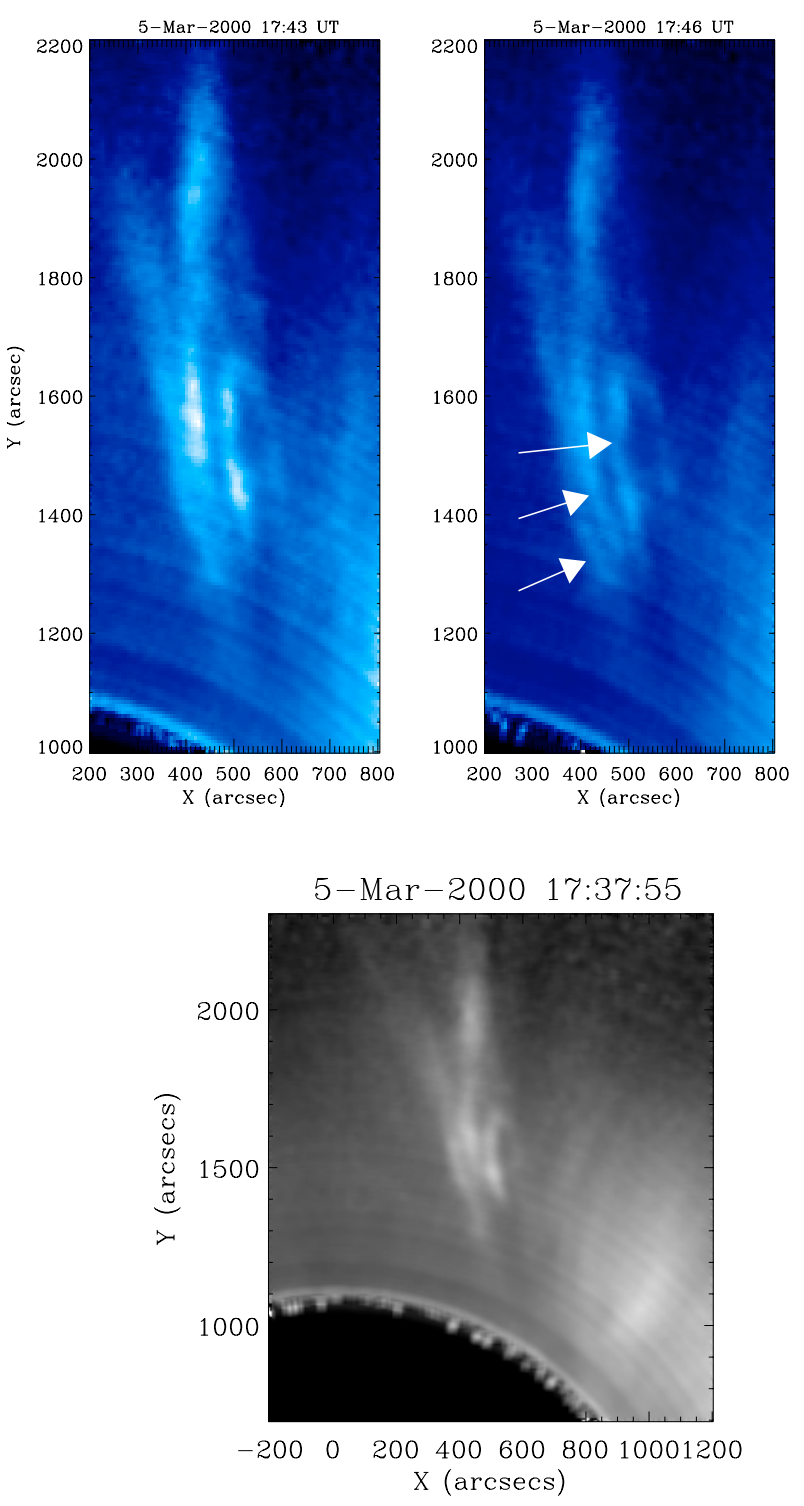

Fig. 7. Images recorded by the Mk4 which was used as an base image in creating Fig. 8.

streak like structures are evident and are marked in the middle panel with arrows. These structures appear quite discrete in the left panel. With time, these streaks diffuse and most probably move down. Figure 8 displays the sequence of base difference images taken by the Mk4 coronameter. Base difference images provide information about the dynamics of features with respect to a fixed image frame. In this case the image recorded at 17:37:55 UT (see Fig. 7) was taken as the base image. We chose this particular image as a base since the leading edge of the downflow started to be clearly discernible at this time. Although Mk4 provides observations with a cadence of about 3 minutes, we only show some selected images in this figure. For the complete sequence see the movie "mk4.mov"5. Three distinct features can be seen in the top left images in Fig. 8. As time passes it appears that the complete structure is breaking into two parts. One moving outwards (see bright feature at 500, 1900 arcsec location in the top right image taken at 17:46 UT) and the other moving downwards (see bright feature at 500, 1400 arcsec in the top right image). The downward moving feature appears

\footnotetext{
${ }^{5}$ Movies are available online.
}

Fig. 6. Absolute intensity images taken by Mk4 white-light coronagraph. The arrows in the middle panel mark the shrinking loops. initially to be blob-like and starts to bifurcate at 17:52:44 UT (more clearly at 17:55:41 UT and afterwards) at a point which corresponds to branches "B1" and "B3" seen in $\mathrm{H} \alpha$ (see Fig. 3). The branching of the downflow was apparent in $\mathrm{H} \alpha$ at 17:54:04 UT becoming more clear at 17:57:09 UT. The bifurcation of the right branch could not be recorded by Mk4 because of the occulting disk. The large dark area, or dimming, that slowly builds up in the center of the image during the sequence is due to a general decrease in the brightness there as the body of the CME and embedded prominence moves. As can be deduced from Fig. 8 and more clearly from the movie "mk4.mov", the dimming area increases in all four directions, but predominantly in the direction of the outflow and downflow. The increase in the dimming area can be interpreted in terms of reconnection as proposed by Shiota et al. (2005) based on MHD modelling.

\section{Height-time and speed-height measurements}

Figure 9 displays the height-time plot for the right branch "B1" of the downflow as measured from the observations recorded by the $\mathrm{H} \alpha$ coronagraph (asterisks), Mk4 coronameter (triangles) and EIT $195 \AA$ (diamonds). Note that the data points from Mk4 are obtained from the base difference images (since in the original images the features are rather diffuse and it is easier to follow the dynamics of features using base difference images) and those for EIT are taken from Paper I. These data points refer to the leading edge of the features. We did not attempt to compare the height-time diagram for other branches as they were not observed with all instruments simultaneously. The height-time diagram for the right branch matches very well for all three instruments except the very first point of EIT. This could be due to an error in the position of obtaining the first EIT data point as it was quite weak and lay right at the edge of the image frame. In general the gas falls more rapidly in the early phases and more slowly in the later phases.

Figure 10 displays the speed-height profiles of the right branch (B1, panel a), middle branch (B2, panel b) and left branch (B3, panel c) as obtained from the $\mathrm{H} \alpha$ observations. The dashed line represents the downward component of the speed and the dotted line indicates the absolute projected speed for the downflows. In order to avoid excessive fluctuations in the speed we 

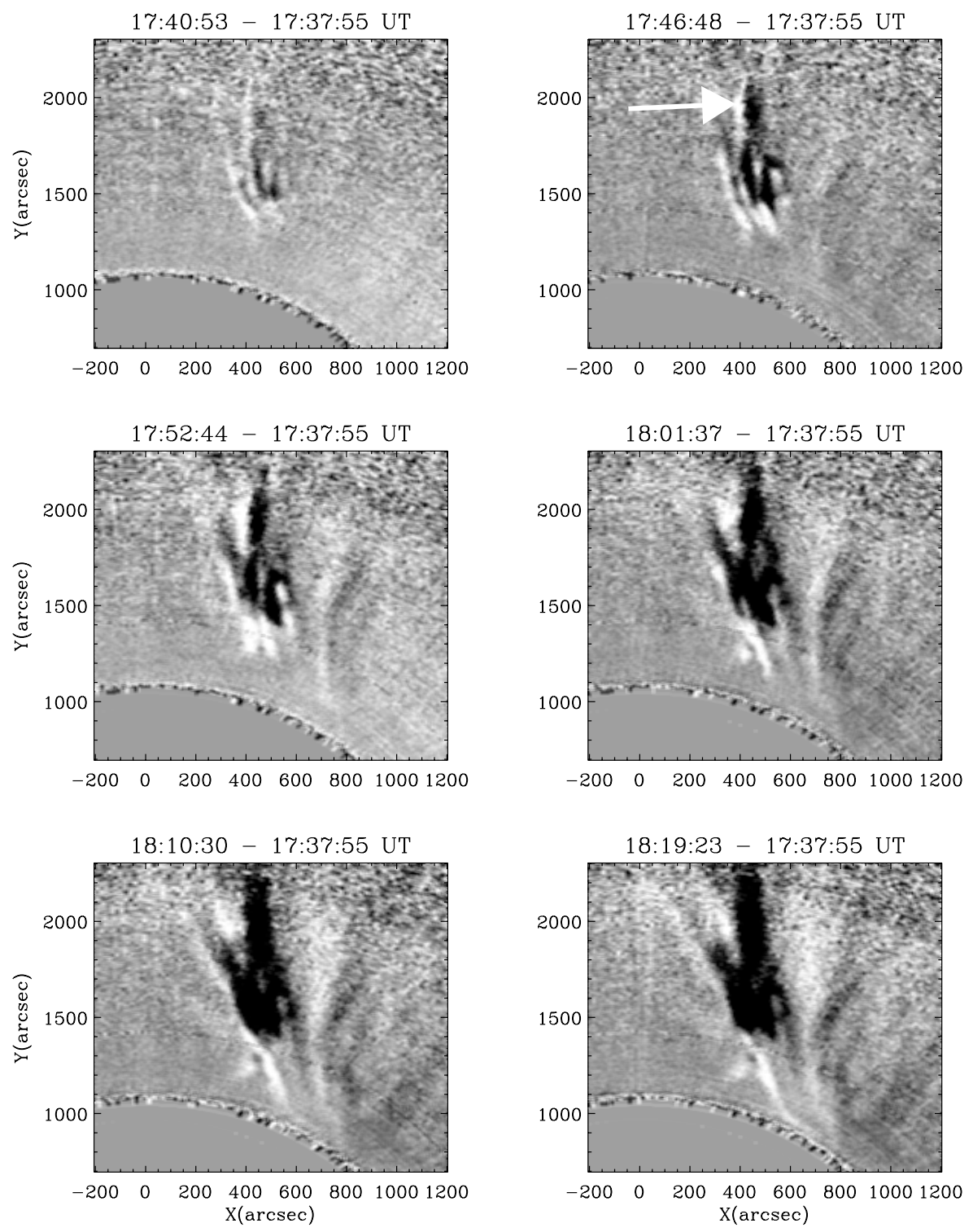

Fig. 8. Base difference images taken by the Mk4 coronameter of the Advanced Coronal Observing System at Mauna Loa Solar Observatory. The arrow in the top right panel locates the outward propagating reconnetion jet. Note that only few images are shown here. For the complete sequence see the movie "mk4.mov" (online only).

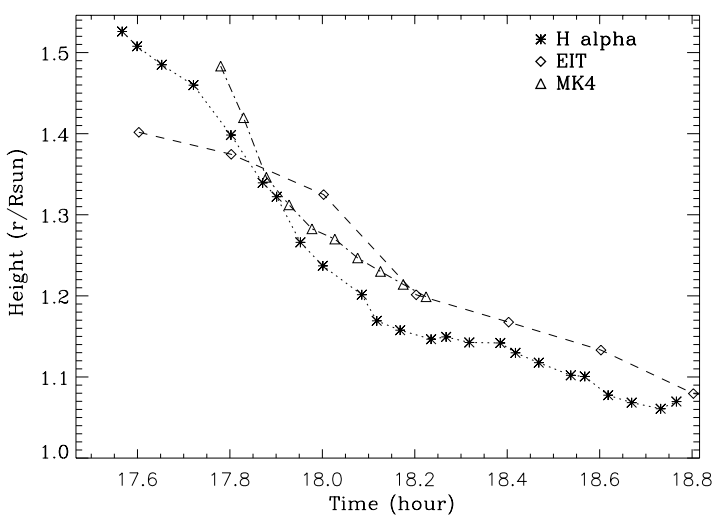

Fig. 9. Height-time plot of the right branch (B1) of the downflow based on observations recorded by the $\mathrm{H} \alpha$ coronagraph (asterisks), Mk4 (triangles) and EIT $195 \AA$ (diamonds). Note that the data points for Mk4 were obtained from base-difference images. EIT data points are taken from Tripathi et al. (2006b).

have applied a smoothing (running mean) to the data points as we are mainly interested in the general trend of speeds rather than their local fluctuations. Note that all the measured speeds represent a lower limit on the real speed, since the direction of motion may lie outside the plane of the sky. The solid lines represent the speed-height profile of a ballistic body falling from a height of $1.6 R_{\odot}$, taking only solar gravitation into account. The free fall height was chosen to be $1.6 R_{\odot}$ as the movie "mk4.mov" reveals that the bifurcation would have happened at around this height. It is evident from Fig. 10 that the right and the left branches of the downflow seen in $\mathrm{H} \alpha$ (panels a and c) started with almost the free-fall speed and were strongly decelerated. However, the middle branch downflow (panel b) had a speed well below free fall. This is clearly due to the fact that this branch bifurcated from the right branch at a time when the latter was already highly decelerated.

Figure 11 displays the speed-height profile for the right branch (B1, panel a) and that of the left branch (B3, panel b) from Mk4. The different lines have the same meaning as in Fig. 10. Recall that the data points for Mk4 were obtained from the base difference images. There are no measurements for the middle branch as it was below the occulting disk of Mk4. The initial speeds measured from the base difference Mk4 images are much higher than those obtained from the $\mathrm{H} \alpha$ absolute 

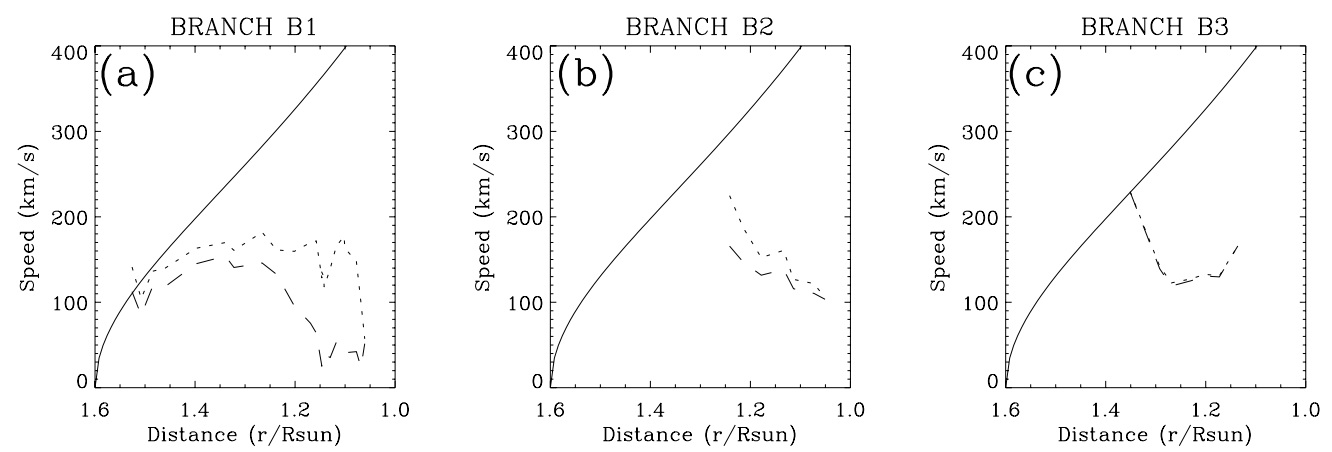

Fig. 10. Speed-height plots for the downflow (B1, right branch: panel a); B2, left branch: panel b); B3, left branch: panel c) based on the H $\alpha$ coronagraph observations. Dashed lines represent the absolute projected speed and dotted lines represent the downward component of the speed. The solid lines represent the free-fall speed profile of a body starting at $1.6 R_{\odot}$. The variation of gravitational acceleration with height was taken into account.

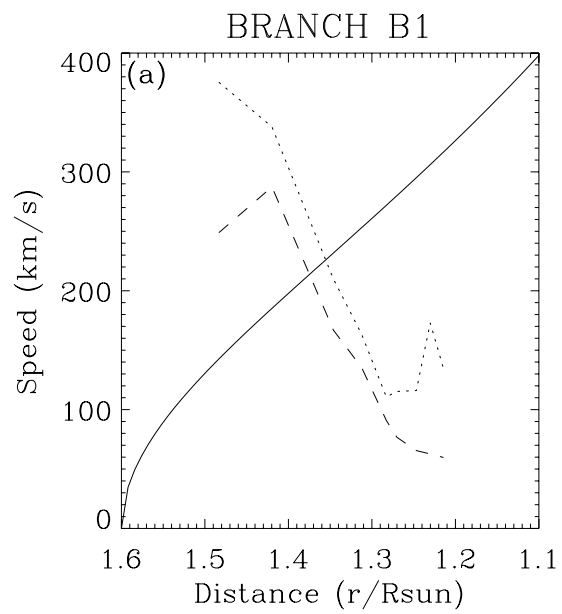

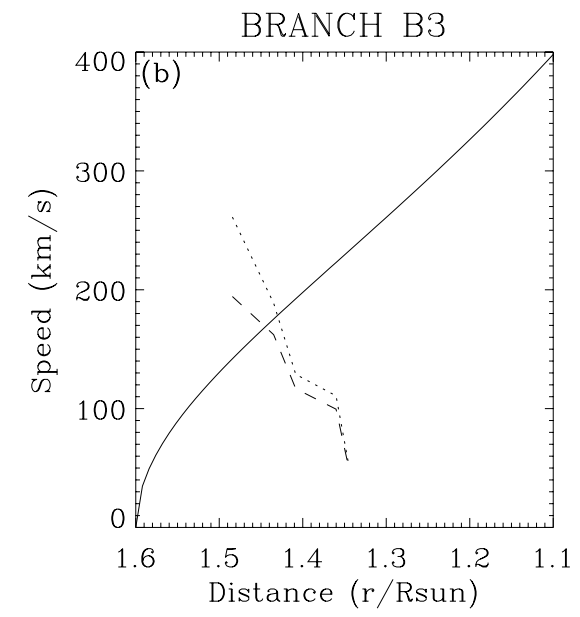

Fig. 11. Speed-height plots for the downflow (B1, right branch: panel a); B3, left branch: panel b) based on the Mk4 coronagraph observations. Dotted lines represent the absolute projected speed and dashed lines represent the downward component of the speed. The solid lines represent the free-fall speed profile of a ballistic body starting at $1.6 R_{\odot}$. The variation of gravitational acceleration with height was taken into account. intensity images. The downflow started with a much higher speed $\left(\approx 380 \mathrm{~km} \mathrm{~s}^{-1}\right)$ than free fall, but then rapidly decelerated to values well below free-fall. These later speeds are in reasonable agreement with those found from $\mathrm{H} \alpha$. The right and left branches show similar speed profiles, although the speed of the left branch downflow is lower than that of the right branch.

Based on the MHD treatment of magnetic reconnection (i.e. Sweet-Parker and Petschek type reconnection), the reconnection outflow speed appears to be roughly equivalent to Alfvén speed. If the total magnetic energy stored at the reconnection location converts into the bulk kinetic energy of the plasma, the outflow speed become equal to the Alfvén speed (Yokoyama et al. 2001). Considering the magnetic field in prominences to be about $20 \mathrm{G}$ (see e.g., Casini et al. 2003) and the density as $10^{10} \mathrm{~cm}^{-3}$ (see e.g., Chang \& Deming 1998), the Alfvén speed is then about $\approx 440 \mathrm{~km} \mathrm{~s}^{-1}$. This is larger than the measured initial speed of the downflow. Taking into account that some of the energy converts into the thermal energy heating the plasma, the initial speed of the downflow appears to be consistent with a reconnection outflow.

\section{Summary and discussion}

We have investigated the multi-wavelength observation taken by SoHO/EIT at $195 \AA$, and the ACOS instruments namely, the PICS $\mathrm{H} \alpha$ coronagraph and the Mk4 white-light K-coronameter in order to study the origin, evolution and characteristics of a bright coronal downflow. The downflow was observed after three prominences simultaneously erupted as part of a CME on 5 March 2000. Although there were three prominence eruptions (two large (P1 and P2) and one small (P3); see Fig. 1 in Paper I), only one CME was detected by LASCO/C2 coronagraph. The two large prominences (P1 and P2) were identified in LASCO/C2 images as the core of the CME (see Fig. 2 in Paper I).

\subsection{Summary of results}

- The downflow is seen in the EIT (195 ^, $171 \AA$, $284 \AA$, $304 \AA$ ), SXT (see Paper I), ACOS H $\alpha$ coronagraph and Mk4 white-light coronameter observations, so that it must comprise multi-thermal plasma.

- The downflows observed by different instruments appears to be approximately co-spatial and co-temporal, although the cool $\mathrm{H} \alpha$ gas lies somewhat below the hotter EIT gas most of the time (see Fig. 9). The larger FOV of the Mk4 coronameter relative to that of the EIT allowed us to determine that the downflow started roughly at $1.6 R_{\odot}$.

- The Mk4 base difference images show a blob-like feature pushed downward at around 17:46 UT which later bifurcated into two branches namely "B1" and "B3" (see Fig. 8, top right panel). Simultaneously, a bright outward moving feature was seen (see Fig. 8, images in the second and third row). For a complete sequence see movie "mk4.mov" (available online).

- The kink in the right branch (branch "B1") of the downflow was seen at 18:12 UT in EIT observations and at 18:05 UT in $\mathrm{H} \alpha$ coronagraph observation. The middle branch (branch 
"B2") of the downflow emanated at the location of the kink. This branch is only seen in $\mathrm{H} \alpha$ observations. In the EIT, the middle branch was not clearly observed, although an bright streak emanating from the kink location is apparent in the running difference images shown in the third image in the first column of Fig. 1.

- The $\mathrm{H} \alpha$ images show many bright streaks which are probably aligned along the magnetic field. At least three can be seen clearly and are labelled "S1", "S2" and "S3" in the image shown in the second row first column in Fig. 3. The Mk4 white light images show a bright shrinking loop-like structures with cusp-shaped loop top (last 3 frames of Fig. 8).

- The $\mathrm{H} \alpha$ observations also reveal that the amount of the downflowing plasma at low temperature increases over most of the time that the downflow was seen. This is suggestive of condensation of plasma from high coronal temperatures. Further the amount of $\mathrm{H} \alpha$ plasma starts to decrease when most of the material drains down to the Sun's surface.

- The height-time plot (see Fig. 9) of the right branch of the downflow shows a similar profile for all three different instruments, with hot plasma (see EIT) lying above the cooler plasma. The height-time profile clearly reveals the kink in space and time. Note that we only produced the height-time plot for the right branch as this was observed with all three instruments simultaneously.

- Speed-height diagrams reveal that the downflow seen in $\mathrm{H} \alpha$ started with almost free-fall speed, but then slowed (Fig. 10). However, Mk4 observations (Fig. 11) show that the initial downflow speed was much higher than the free-fall speed. We note that Mk4 (white-light coronagraph) is sensitive to plasma at all temperatures, so the hot plasma might be moving faster than the cool plasma.

\subsection{Discussion}

Let us consider possible explanations for the origin of the downflow and its characteristics. One possibility is that the downflow is composed of material that could not reach the escape speed and overcome the solar gravitational field. Under this scenario the speed of the downflowing plasma should not exceed that of free fall. It can be lower, since lower lying, possibly still upward moving, plasma would slow down the downflowing plasma. The speed obtained from the $\mathrm{H} \alpha$ observations is comparable to that of free-fall or lies below it. However, the initial downflow speed obtained from the Mk4 recording lies far in excess of the freefall speed. Note that the speeds are measured in the plane of the sky so that they are lower limits. Furthermore, the speeds derived from the Mk4 observations have the advantage over those from the $\mathrm{H} \alpha$ or the EIT observations because the Mk4 observations are not temperature sensitive. Therefore the derived data points based on the Mk4 observations are not temperature biased. There may, however, be an enhanced uncertainty due to the fact that we are analyzing base difference images of Mk4 data. We note that, the downflow was observed in EIT (1.5 MK) and SXT (2$5 \mathrm{MK}$ ) images (see Paper I). This is suggestive of plasma heating which is highly unlikely if the downflow is simply due to deceleration/acceleration by the solar gravitational field.

Another interpretation is that while erupting the prominences pass through a kink instability. The development of a kink in prominences during the eruption phase has been observed (e.g., Williams et al. 2005; Rust \& LaBonte 2005) and theoretically modelled (e.g., Török \& Kliem 2005; Fan 2005). The kink instability in the prominence during eruption can explain the cusp-shaped structure formation which is seen in the images taken by the $\mathrm{H} \alpha$ and the Mk4 coronagraphs as well as the EIT. Furthermore, during the eruption the helical field lines, originally holding the prominence at the bottom, get stretched and the material sitting at the bottom of the flux rope drains down along the legs of the flux rope. This interpretation poses similar problems to the earlier one, such as the plasma heating and the high speed of the downflow with respect to free-fall.

Let us now consider the interpretation put forward by Gilbert et al. (2000, 2001, based on the MLSO H $\alpha$ coronagraph observations) and later Gibson \& Fan (2006a,b, based on 3D MHD simulations). These authors proposed that during the eruption of a pre-existing flux rope, reconnection takes place internally and the flux rope breaks into two. The outer part propagates along with the CME and the lower part falls back to the Sun's surface. The basic observables predicted on the basis of the above model would be the simultaneous presence of an X-type structure in the corona, a three part structured CME and the downflowing plasma (Tripathi et al. 2007). One of the most important and plausible signatures of the reconnection would be heating of plasma emanating from the reconnection locations (X-type location) as well as reconnection jets. This implies that the speed of plasma emanating from the reconnection location and flowing towards the Sun's surface would be significantly higher than the free fall speed especially at high temperature. This speed would either grow or decrease depending on the amount of material below the location of reconnection.

Based on the LASCO/C2 and $\mathrm{C} 3$ observations we confirmed that the associated CME was comprised of a bright front, dark cavity and a bright core - representing the prominence material (see Paper I). Also, the downflowing plasma in the EIT and the SXT was bright, implying that the temperature of at least a part of the downflowing plasma can be as high as 4-5 MK or even 20 MK (Tripathi et al. 2006a), providing strong evidence of plasma heating (see Paper I). However, due to the restricted FOV of the EIT and the SXT and lack of observations from 1.5-2.5 $R_{\odot}$, we were not able to locate the precise reconnection point, reconnection jet and estimate the speed of the downflowing plasma in the early phase. The Mk4 and the $\mathrm{H} \alpha$ data helped us to find the location of reconnection because of the larger FOV of these instruments. Based on the Mk4 observations we have a more complete picture of the reconnection such that the reconnection jet propagates outwards (see movie 'mk4.mov') and the initial speed of the downflowing plasma is substantially higher than that of free-fall (see Figs. 8, 11). Furthermore, the whitelight base difference images show that the dimming area increases at the location where the downflow seems to start. The increase in the dimming area provides further strong evidence of reconnection as described by Shiota et al. (2005).

Although this interpretation explains most of the characteristics of the observed downflow, it still poses a problem concerning the reason for approximately co-spatial and co-temporal observation of multi-thermal plasma such as in the $\mathrm{H} \alpha$ (cool material) and EIT (all four channels) and SXT (hot material). There could be different possibilities. First, this could be due to the fact that cooler plasma (lower part of the prominence) seen in the $\mathrm{H} \alpha$ is still rising when reconnection occurs further up. Because of the reconnection, the plasma is stopped and slowly starts to fall down. In this scenario material would not be heated to the temperatures needed to make it visible in the EIT and the SXT. Another possible explanation is that the EIT and the $\mathrm{H} \alpha$ observations indicate a dense flux tube-like structure. The highly dense plasma in the inner part of the flux tube is either cold to start with, or cools down faster than the outer part, as the radiative cooling time is proportional to $n_{\mathrm{e}}^{2}$. This interpretation also 
explains the fact the $\mathrm{H} \alpha$ downflow in the left branch is highly localised and seen at locations where the EIT downflow is brightest. Moreover, the $\mathrm{H} \alpha$ downflows are thinner than those in EIT. On the other hand, it may well be that the downflow is comprised of multi-thermal unresolved magnetic strands. The above two interpretations are also supported by the fact that the total intensity of the masked region increases over most of the time when the downflow is seen (see Fig. 5). This increase is basically due to the enhancement of the downflowing material, which means that the plasma that was at higher temperature cools down to temperatures sensitive to the $\mathrm{H} \alpha$ observations. Later on the total intensity starts to decrease when most of the cool material is drained down on to the Sun's surface and there are no hot material left to cool down.

Despite the fact that we can explain the origin and characteristics of the observed downflow described in this paper and Paper I, the question remains as to why such downflows are rare event?. We also looked at five more $\mathrm{H} \alpha$ observations from MLSO. Draining of the plasma along the legs of erupting prominences was a common phenomenon in the $\mathrm{H} \alpha$ observations for all those five events. These downflows were also seen in the Mk4 observations. However, there were no corresponding signatures in the EIT observations. Also there was no evidence of formation of an X-type structure such as a cusp in the corona. This suggest that reconnection associated with the heating of the prominence gas to coronal temperatures is not an entirely common occurrence.

We note that this is the first observation of its kind and demands further study and a deeper understanding. In order to carry out this kind of study we would require a wide temperature coverage with very high time resolution data over a large field of view. In the future, observations from the Hinode satellite combined with those from the Solar Terrestrial Relation Observatory (STEREO) and later the Solar Dynamics observatory (SDO) may provide a unique opportunity to study these phenomena in more detail.

Acknowledgements. We acknowledge an anonymous referee for comments which certainly improved the quality of the manuscript. DT and HEM acknowledge support from STFC. DW was supported by Air Force Research Lab Contract FA8718-06-C-0015. DT would like to thank Sarah Gibson for many useful discussions and comments. We thank the SoHO-EIT and HAO teams for providing the data and also Joan Burkepile for her help in explaining the instruments. SoHO is a mission of international collaboration between ESA and NASA.

\section{References}

Asai, A., Yokoyama, T., Shimojo, M., \& Shibata, K. 2004, ApJ, 605, L77 Birn, J., Forbes, T. G., \& Hesse, M. 2006, ApJ, 645, 732

Brueckner, G. E., Howard, R. A., Koomen, M. J., et al. 1995, Sol. Phys., 162, 357

Casini, R., López Ariste, A., Tomczyk, S., \& Lites, B. W. 2003, ApJ, 598, L67 Chang, E. S., \& Deming, D. 1998, Sol. Phys., 179, 89

de Groof, A., Berghmans, D., van Driel-Gesztelyi, L., \& Poedts, S. 2004, A\&A, 415,1141

de Groof, A., Bastiaensen, C., Müller, D. A. N., Berghmans, D., \& Poedts, S. 2005, A\&A, 443, 319

Delaboudiniere, J.-P., Artzner, G. E., Brunaud, J., et al. 1995, Sol. Phys., 162, 291

Domingo, V., Fleck, B., \& Poland, A. I. 1995, Sol. Phys., 162, 1

Elmore, D. F., Burkepile, J. T., Darnell, J. A., Lecinski, A. R., \& Stanger, A. L. 2003, in Polarimetry in Astronomy, ed. S. Fineschi, Proc. SPIE., 66 Fan, Y. 2005, ApJ, 630, 543

Gibson, S. E., \& Fan, Y. 2006a, J. Geophys. Res. (Space Physics), 12103

Gibson, S. E., \& Fan, Y. 2006b, ApJ, 637, L65

Gilbert, H. R., Holzer, T. E., Burkepile, J. T., \& Hundhausen, A. J. 2000, ApJ, 537,503

Gilbert, H. R., Holzer, T. E., \& Burkepile, J. T. 2001, ApJ, 549, 1221

Handy, B. N., Acton, L. W., Kankelborg, C. C., et al. 1999, Sol. Phys., 187, 229

Innes, D. E., McKenzie, D. E., \& Wang, T. 2003a, Sol. Phys., 217, 267

Innes, D. E., McKenzie, D. E., \& Wang, T. 2003b, Sol. Phys., 217, 247

Lin, J., \& Forbes, T. G. 2000, J. Geophys. Res., 105, 2375

Manchester, IV, W., Gombosi, T., DeZeeuw, D., \& Fan, Y. 2004, ApJ, 610, 588

McKenzie, D. E. 2000, Sol. Phys., 195, 381

McKenzie, D. E., \& Hudson, H. S. 1999, ApJ, 519, L93

Moses, D., Clette, F., Delaboudinière, J.-P., et al. 1997, Sol. Phys., 175, 571

Rust, D. M., \& LaBonte, B. J. 2005, ApJ, 622, L69

Sheeley, Jr., N. R., \& Wang, Y.-M. 2002, ApJ, 579, 874

Shiota, D., Isobe, H., Chen, P. F., et al. 2005, ApJ, 634, 663

Török, T., \& Kliem, B. 2005, ApJ, 630, L97

Tripathi, D., Del Zanna, G., Mason, H. E., \& Chifor, C. 2006a, A\&A, 460, 53

Tripathi, D., Solanki, S. K., Schwenn, R., et al. 2006b, A\&A, 449, 369

Tripathi, D., Gibson, S. E., Qui, J., et al. 2007, A\&A, in preparation

Tsuneta, S., Acton, L., Bruner, M., et al. 1991, Sol. Phys., 136, 37

Wang, Y.-M., Sheeley, N. R., Howard, R. A., St. Cyr, O. C., \& Simnett, G. M. 1999, Geophys. Res. Lett., 26, 1203

Wilhelm, K., Curdt, W., Marsch, E., et al. 1995, Sol. Phys., 162, 189

Williams, D. R., Török, T., Démoulin, P., van Driel-Gesztelyi, L., \& Kliem, B. 2005, ApJ, 628, L163

Yokoyama, T., Akita, K., Morimoto, T., Inoue, K., \& Newmark, J. 2001, ApJ, 546, L69 